\title{
Event-by-Event Fluctuations in Ultrarelativistic Heavy-Ion Collisions
}

\author{
Gordon Baym*+ and Henning Heiselberg ${ }^{+}$ \\ * Department of Physics, University of Illinois at Urbana-Champaign, 1110 W. Green St., Urbana, Illinois 61801, USA \\ ${ }^{+}$NORDITA, Blegdamsvej 17, DK-2100 Copenhagen Ø, Denmark
}

(Submitted to Physics Letters B, 15 May 1999)

\begin{abstract}
Motivated by forthcoming experiments at RHIC and LHC, we study event-by-event fluctuations in ultrarelativistic heavy-ion collisions in participant nucleon as well as thermal models. The calculated physical observables, including multiplicity, kaon to pion ratios, and transverse momenta agree well with recent NA49 data at the SPS, and indicate that such studies do not yet reveal the presence of new physics. Finally, we present a simple model of how a first order phase transition can be signaled by very large fluctuations.
\end{abstract}

PACS numbers: 25.75+r, 24.85.+p, 25.70.Mn, 24.60.Ky, 24.10.-k

Keywords: Relativistic heavy-ion collisions; Fluctuations; Event-by-Event Analyses

Central ultrarelativistic collisions at RHIC and LHC are expected to produce at least $\sim 10^{4}$ particles, and thus present one with the remarkable opportunity to analyze, on an event-by-event basis, fluctuations in physical observables such particle multiplicities, transverse momenta, correlations and ratios. Analysis of single events with large statistics can reveal very different physics than studying averages over a large statistical sample of events. The use of Hanbury Brown-Twiss correlations to extract the system geometry is a familiar application of event-by-event fluctuations in nuclear collisions [1], and elsewhere, e.g, in sonoluminesence [2]. The power of this tool has been strikingly illustrated in study of interference between Bose-Einstein condensates in trapped atomic systems [3]. Recently NA49 has presented a prototypical event-by-event analysis of fluctuations in central $\mathrm{Pb}+\mathrm{Pb}$ collisions at $158 \mathrm{GeV}$ per nucleon at the SPS, which produce more than a thousand particles per event 顿.

Studying event-by-event fluctuations in ultrarelativistic heavy ion collisions to extract new physics was proposed in a series of papers by the authors and coworkers [5-7], in which the analysis of transverse energy fluctuations in central collisions [8] was used to extract evidence within the binary collision picture for color, or cross-section, fluctuations. More recent theoretical papers have focussed on different aspects of these fluctuations, such as searching for evidence for thermalization [4,9], and critical fluctuations at the QCD phase transition [11, 12].

In order to be able to extract new physics associated with fluctuations, it is necessary to understand the role of expected statistical fluctuations. Our aim here is to study the sources of these fluctuations in collisions. As we shall see, the current NA49 data can be essentially understood on the basis of straightforward statistical arguments. Expected sources of fluctuations include impact parameter fluctuations, fluctuations in the number of primary collisions, and the results of such collisions, nuclear deformations [8], effects of rescattering of secon- daries, and qcd color fluctuations. Since fluctuations in collisions are sensitive to the amount of rescattering of secondaries taking place, we discuss in detail two limiting cases, the participant or "wounded nucleon model" (WNM), in which one assumes that particle production occurs in the individual participant nucleons and rescattering of secondaries is ignored, and the thermal limit in which scatterings bring the system into local thermal equilibrium. Whether rescatterings increase relative fluctuations through greater production of multiplicity, transverse momenta, etc., or decrease fluctuations by involving a greater number of degrees of freedom, is not immediately obvious. Indeed VENUS simulations 13. showed that rescattering had negligible effects on transverse energy fluctuations. As we shall see, both models give similar results for multiplicity fluctuations. In the wounded nucleon model fluctuations arise mainly from multiplicity fluctuations for each participant and from impact parameter fluctuations. Limited acceptance also influences the observed fluctuations. We calculate in detail statistical fluctuations in multiplicity, $\mathrm{K} / \pi$ ratios, and transverse momentum. Finally, we show in a simple model how first order phase transitions are capable of producing very significant fluctuations.

Multiplicity fluctuations: Let us first calculate fluctuations in the participant model, which appears to describe well physics at SPS energies [4]. In this picture

$$
N=\sum_{i}^{N_{p}} n_{i}
$$

where $N_{p}$ is the number of participants and $n_{i}$ is the number of particles produced in the acceptance by participant $i$. In the absence of correlations between $N_{p}$ and $n$, the average multiplicity is $\langle N\rangle=\left\langle N_{p}\right\rangle\langle n\rangle$. For example, NA49 measures charged particles in the rapidity region $4<y<5.5$ and finds $\langle N\rangle \simeq 270$ for central $\mathrm{Pb}+\mathrm{Pb}$ collisions. Finite impact parameters $(b \lesssim 3.5 \mathrm{fm})$ as well as surface diffuseness reduce the number of participants 
from the total number of nucleons $2 A$ to $\left\langle N_{p}\right\rangle \simeq 350$ estimated from Glauber theory; thus $\langle n\rangle \simeq 0.77$. Squaring Eq. (11) assuming $\left\langle n_{i} n_{j}\right\rangle=\left\langle n_{i}\right\rangle\left\langle n_{j}\right\rangle$ for $i \neq j$, we find the multiplicity fluctuations

$$
\omega_{N}=\omega_{n}+\langle n\rangle \omega_{N_{p}}
$$

where in general we write

$$
\sigma(y)=\left\langle y^{2}\right\rangle-\langle y\rangle^{2} \equiv\langle y\rangle \omega_{y}
$$

for any stochastic variable $y$.

A major source of multiplicity fluctuations per participant, $\omega_{n}$, is the limited acceptance. While each participant produces $\nu$ charged particles, only a smaller fraction $f=\langle n\rangle /\langle\nu\rangle$ are accepted. Without carrying out a detailed analysis of the acceptance, one can make a simple statistical estimate assuming that the particles are accepted randomly, in which case $n$ is binomially distributed with $\sigma(n)=\nu f(1-f)$ for fixed $\nu$. Including fluctuations in $\nu$ we obtain, similarly to Eq. (2),

$$
\omega_{n}=1-f+f \omega_{\nu} .
$$

In NN collisions at SPS energies, the charged particle multiplicity is $\sim 7.3$ and $\omega_{\nu} \simeq 1.9$ [14]; thus $\langle\nu\rangle \simeq 3.7$ and $f \simeq 0.21$ for the NA49 acceptance. Consequently, we find from Eq. (4) that $\omega_{n} \simeq 1.2$.

As a consequence of nuclear correlations, which strongly reduce density fluctuations in the colliding nuclei, the fluctuations $\omega_{N_{p}(b)}$ in $N_{p}$ are very small for fixed impact parameter $b$ [7]. Almost all nucleons in the nuclear overlap volume collide and participate. [By contrast, the fluctuations in the number of binary collisions is non-negligible.] Cross section fluctuations play a small role in the WNM [7]. Fluctuations in the number of participants can arise when the target nucleus is deformed, since the orientations of the deformation axes vary from event to event [15]. The fluctuations, $\omega_{N_{p}}$, in the number of participants are dominated by the varying impact parameters selected by the experiment. In the NA49 experiment, for example, the zero degree calorimeter selects the $5 \%$ most central collisions, corresponding to impact parameters smaller than a centrality cut on impact parameter, $b_{c} \simeq 3.5 \mathrm{fm}$. We have

$$
\omega_{N_{p}}\left\langle N_{p}\right\rangle=\frac{1}{\pi b_{c}^{2}} \int_{0}^{b_{c}} d^{2} b N_{p}(b)^{2}-\left\langle N_{p}\right\rangle^{2}
$$

where $\left\langle N_{p}\right\rangle=\left(1 / \pi b_{c}^{2}\right) \int_{0}^{b_{c}} d^{2} b N_{p}(b)$. The number of participants for a given centrality, calculated in [17, can be approximated by $N_{p}(b) \simeq N_{p}(0)(1-b / 2 R)$ for $0 \leq b \lesssim 3.5 \mathrm{fm}$; thus

$$
\omega_{N_{p}}=\frac{N_{p}(0)}{18}\left(\frac{b_{c}}{2 R}\right)^{2} .
$$

For NA49 $\mathrm{Pb}+\mathrm{Pb}$ collisions with $N_{p}(0) \simeq 400$ and $\left(b_{c} / 2 R\right)^{2} \simeq 5 \%$ we find $\omega_{N_{p}} \simeq 1.1$. Impact parameter fluctuations are thus important even for the centrality trigger of NA49. Varying the centrality cut or $b_{c}$ to control such impact parameter fluctuations (6) should enable one to extract better any more interesting intrinsic fluctuations. Recent WA98 analyses confirm that fluctuations in photons and pions grow approximately linearly with the centrality cut 16]. The Gaussian multiplicity distribution found in central collisions changes for minimum bias to a plateau-like distribution [8].

Calculating $\omega_{N}$ for the NA49 parameters, we find from Eq. (2), $\omega_{N} \simeq 1.2+(0.77)(1.1)=2.0$, in good agreement with experiment, which measures a multiplicity distribution $\propto \exp \left[-(N-\langle N\rangle)^{2} / 2\langle N\rangle \omega_{N}^{e x p}\right]$, where $\omega_{M}^{\exp }$ is of order 2.01 近.

Let us now consider, in the opposite limit of considerable rescattering, fluctuations in thermal models. In a gas in equilibrium, the mean number of particles per bosonic mode $n_{a}$ is given by

$$
\left\langle n_{a}\right\rangle=\left(\exp \left(E_{a} / T\right)-1\right)^{-1}
$$

with fluctuations

$$
\omega_{n_{a}}=1+\left\langle n_{a}\right\rangle .
$$

The total fluctuation in the multiplicity, $N=\sum_{a} n_{a}$, is

$$
\omega_{N}^{B E}=1+\sum_{a}\left\langle n_{a}\right\rangle^{2} / \sum_{a}\left\langle n_{a}\right\rangle .
$$

If the modes are taken to be momentum states, the resulting fluctuations are $\omega_{N}^{B E}=\zeta(2) / \zeta(3)=1.37$ for massless particles, while for pions at temperature $T=150 \mathrm{MeV}$ $\omega_{N}^{B E}=1.11[18]$.

Resonances add to fluctuations in the thermal limit whereas they are implicitly included in the WNM fluctuations. In high energy nuclear collisions, resonance decays such as $\rho \rightarrow 2 \pi, \omega \rightarrow 3 \pi$, etc., lead to half or more of the pion multiplicity. Only a small fraction $r \simeq 10 \%$ produce two charged particles in a thermal hadron gas 19] or in RQMD [20]. Including such resonance fluctuations in the BE fluctuations gives, similarly to Eq. (2),

$$
\omega_{N}^{B E+R}=r \frac{1-r}{1+r}+(1+r) \omega_{N}^{B E}
$$

With $r \simeq 0.1$ we obtain $\omega_{N}^{B E+R} \simeq 1.3$. If not all of the decay particles fall into the NA49 acceptance the fluctuations from resonances will be reduced. In [12] the estimated effect of resonances is about twice ours: $\omega_{N} \simeq 1.5$, not including impact parameter fluctuations.

Fluctuations in the effective collision volume add a further term $\langle N\rangle \sigma(V) /\langle V\rangle^{2}$ to $\omega_{N}^{B E+R}$. Assuming that the volume scales with the number of participants, $\omega_{V} /\langle V\rangle \simeq$ $\omega_{N_{p}} /\left\langle N_{p}\right\rangle$, we find from Eq. (2) that $\omega_{N}=\omega_{N}^{B E+R}+$ $\langle n\rangle \omega_{N_{p}} \simeq 2.1$, again consistent with the NA49 data. Because of the similarity between the magnitudes of the thermal and WNM multiplicity fluctuations, the present 
measurements cannot distinguish between these two limiting pictures.

Kaon/pion ratio: To second order in the fluctuations of the numbers of $\mathrm{K}$ and $\pi$, we have

$$
\langle K / \pi\rangle=\frac{\langle K\rangle}{\langle\pi\rangle}\left(1+\frac{\omega_{\pi}}{\langle\pi\rangle}-\frac{\langle K \pi\rangle-\langle K\rangle\langle\pi\rangle}{\langle K\rangle\langle\pi\rangle}\right) .
$$

The corresponding fluctuations in $\langle K / \pi\rangle$ are given by

$$
D^{2} \equiv \frac{\omega_{K / \pi}}{\langle K / \pi\rangle}=\frac{\omega_{K}}{\langle K\rangle}+\frac{\omega_{\pi}}{\langle\pi\rangle}-2 \frac{\langle K \pi\rangle-\langle K\rangle\langle\pi\rangle}{\langle K\rangle\langle\pi\rangle} .
$$

The fluctuations in the kaon to pion ratio is dominated by the fluctuations in the number of kaons alone. The third term in Eq. (12) includes correlations between the number of pions and kaons. It contains a negative part from volume fluctuations, which removes the volume fluctuations in $\omega_{K}$ and $\omega_{\pi}$ since such fluctuations cancel in any ratio. In the NA49 data [4] the average ratio of charged kaons to charged pions is $\langle K / \pi\rangle=0.18$ and $\langle\pi\rangle \simeq 220$. Excluding volume fluctuations, we take $\omega_{K} \simeq \omega_{\pi} \simeq 1.2-1.3$ as discussed above. The first two terms in Eq. (12) then yield $D \simeq 0.20$ in good agreement with preliminary measurements $D=0.23$ [ [ 1 . Thus at this stage the data gives no evidence for correlated production of $\mathrm{K}$ and $\pi$, as described by the final term in Eq. (12), besides volume fluctuations. The similar fluctuations in mixed event analyses $D_{\text {mixed }}=0.208$ [4] confirm this conclusion.

Transverse momentum fluctuations: The total transverse momentum per event

$$
P_{t}=\sum_{i=1}^{N} p_{t, i}
$$

is very similar to the transverse energy, for which fluctuations have been studied extensively [8,6]. The mean transverse momentum and inverse slopes of distributions generally increase with centrality or multiplicity. Assuming that $\alpha \equiv d \log \left(\left\langle p_{t}\right\rangle_{N}\right) / d \log N$ is small, as is the case for pions [21], the average transverse momentum per particle for given multiplicity $N$ is to leading order

$$
\left\langle p_{t}\right\rangle_{N}=\left\langle p_{t}\right\rangle(1+\alpha(N-\langle N\rangle) /\langle N\rangle) .
$$

where $\left\langle p_{t}\right\rangle$ is the average over all events of the single particle transverse momentum. With this parametrization, the average total transverse momentum per particle in an event obeys $\left\langle P_{t} / N\right\rangle=\left\langle p_{t}\right\rangle$. When the transverse momentum is approximately exponentially distributed with inverse slope $T$ in a given event, $\left\langle p_{t, i}\right\rangle=2 T$, and $\sigma\left(p_{t, i}\right)=2 T^{2}=\left\langle p_{t}\right\rangle^{2} / 2$. This latter fluctuation is in principle dependent on multiplicity, but as a higher order effect, we ignore it in the following.

The total transverse momentum per particle in an event has fluctuations

$$
\begin{aligned}
\langle N\rangle \sigma\left(P_{t} / N\right) & =\sigma\left(p_{t}\right)+\alpha^{2}\left\langle p_{t}\right\rangle^{2} \omega_{N} \\
& +\left\langle\frac{1}{N} \sum_{i \neq j}\left(p_{t, i} p_{t, j}-\left\langle p_{t}\right\rangle^{2}\right)\right\rangle .
\end{aligned}
$$

The three terms on the right are respectively:

i) The individual fluctuations $\sigma\left(p_{t, i}\right)=\left\langle p_{t, i}^{2}\right\rangle-\left\langle p_{t}\right\rangle^{2}$, the main term. In the NA49 data, $\left\langle p_{t}\right\rangle=377 \mathrm{MeV}$ and $\langle N\rangle=270$. From Eq. (15) we thus obtain $\left(\sigma\left(P_{t} / N\right)^{1 / 2} /\left\langle p_{t}\right\rangle \simeq 1 / \sqrt{2\langle N\rangle}=4.3 \%\right.$, which accounts for most of the experimentally measured fluctuation $4.65 \%$. The data contains no indication of intrinsic temperature fluctuations in the collisions.

ii) Effects of correlations between $p_{t}$ and $N$, which are suppressed with respect to the first term by a factor $\sim \alpha^{2}$. In NA49 the multiplicity of charged particles is mainly that of pions for which $T \simeq\left\langle p_{t}\right\rangle / 2$ increases little compared with pp collisions, and $\alpha \simeq 0.05-0.1$. Thus, these correlations are small for the NA49 data. However, for kaons and protons, $\alpha$ can be an order of magnitude larger as their distributions are strongly affected by the flow observed in central collisions [21].

iii) Correlations between transverse momenta of different particles in the same event. In the WNM the momenta of particles originating from the same participant are correlated. In Lund string fragmentation, for example, a quark-antiquark pair is produced with the same $p_{t}$ but in opposite direction. The average number of pairs of hadrons from the same participant is $\langle n(n-1)\rangle$ and therefore the latter term in Eq. (15) becomes $(\langle n(n-1)\rangle\langle n\rangle)\left(\left\langle p_{t, i} p_{t, j \neq i}\right\rangle-\left\langle p_{t}\right\rangle^{2}\right)$. To a good approximation, $n$ is Poisson distributed, i.e., $\langle n(n-1)\rangle /\langle n\rangle=$ $\langle n\rangle$, equal to 0.77 for the NA49 acceptance, so that this latter term becomes $\simeq\left(\left\langle p_{t, i} p_{t, j \neq i}\right\rangle-\left\langle p_{t}\right\rangle^{2}\right)$. The momentum correlation between two particles from the same participant is expected to be a small fraction of $\sigma\left(p_{t, i}\right)$.

To quantify the effect of rescatterings, Ref. [9] suggested studying the differences in $\langle N\rangle \sigma\left(P_{t} / N\right)$ and $\sigma\left(p_{t}\right)$ via the quantity

$$
\Phi\left(p_{t}\right) \simeq \sqrt{\langle N\rangle \sigma\left(P_{t} / N\right)}-\sqrt{\sigma\left(p_{t, i}\right)} .
$$

As we see from Eq. (15), in the applicable limit that the second and third terms are small,

$$
\Phi\left(p_{t}\right) \simeq \frac{1}{\sqrt{\sigma\left(p_{t, i}\right)}}\left(\alpha^{2}\left\langle p_{t}\right\rangle^{2} \omega_{N}+\left(\left\langle p_{t, i} p_{t, j \neq i}\right\rangle-\left\langle p_{t}\right\rangle^{2}\right)\right)
$$

In the Fritiof model, based on the WNM with no rescatterings between secondaries, one finds $\Phi\left(p_{t}\right) \simeq 4.5 \mathrm{MeV}$. In the thermal limit the correlations in Eq. (16) should vanish for classical particles but the interference of identical particles (HBT correlations) contribute to these correlations by $\sim 6.5 \mathrm{MeV} 10$ and slightly reduced by resonances. The NA49 experimental value, $\Phi\left(p_{t}\right)=5 \mathrm{MeV}$ (corrected for two-track resolution) seems to favor the thermal limit [4]. Note however that with $\alpha \simeq 0.05-0.1$, the second term on the right side of Eq. (17) alone leads 
to $\Phi \simeq 1-4 \mathrm{MeV}$, i.e., the same order of magnitude. If $\left(\left\langle p_{t, i} p_{t, j \neq i}\right\rangle-\left\langle p_{t}\right\rangle^{2}\right)$ is not positive, then one cannot a priori rule out that the smallness of $\Phi\left(p_{t}\right)$ does not arise from a cancellation of this term with $\alpha^{2}\left\langle p_{t}\right\rangle^{2} \omega_{N}$, rather than from thermalization.

First order phase transitions can lead to rather large fluctuations in physical quantities. Thus, detection of enhanced fluctuations, beyond the elementary statistical ones considered to this point, could signal the presence of such a transition. For example, matter undergoing a transition from chirally symmetric to broken chiral symmetry could, when expanding, supercool and form droplets, resulting in large multiplicity versus rapidity fluctuations [22]. Let us imagine that $N_{D}$ droplets fall into the acceptance, each producing $n$ particles, i.e., $\langle N\rangle=\left\langle N_{D}\right\rangle\langle n\rangle$. The corresponding multiplicity fluctuation can be computed analogously to Eq. (2)

$$
\omega_{N}=\omega_{n}+\langle n\rangle \omega_{N_{D}} .
$$

As in Eq. (4), we expect $\omega_{n} \sim 1$. However, unlike the case of participant fluctuations, the second term in (18) can lead to huge multiplicity fluctuations when only a few droplets fall into the acceptance; in such a case, $\langle n\rangle$ is large and $\omega_{N_{D}}$ of order unity. The fluctuations from droplets depends on the total number of droplets, the spread in rapidity of particles from a droplet, $\delta y \sim$ $\sqrt{T / m_{t}}$, as well as the experimental acceptance in rapidity, $\Delta y$. When $\delta y \ll \Delta y$ and the droplets are binomially distributed in rapidity, $\omega_{N_{D}} \simeq 1-\Delta y / y_{\text {tot }}$, which can be a significant fraction of unity.

In the extreme case where none or only one droplet falls into the acceptance with equal probability, we have $\omega_{N_{D}}=1 / 2$ and $\langle n\rangle=2\langle N\rangle$. The resulting fluctuation is $\omega_{N} \simeq\langle N\rangle$, which is more than two orders of magnitude larger than the expected value of order unity as currently measured in NA49. This simple example clearly demonstrates the importance of event-by-event fluctuations accompanying phase transitions, and illustrates how monitoring such fluctuations versus centrality becomes a promising signal, in the upcoming RHIC experiments, for the onset of a transition.

This work was initiated at the 13th Nordic Meeting on Intermediate and High Energy Nuclear Physics, Graeftaavallen, Sweden, and supported in part by National Science Foundation Grants No. PHY94-21309 and PHY9800978. We are grateful to A.D. Jackson for discussions, and Volker Koch and Sergei Voloshin for helpful comments.
[1] U. Heinz and B.V. Jacak, Ann. Rev. Nucl. Part. Sci. 49 (1999), and references therein.

[2] S. Trentalange and S.U. Pandey, J. Acoust. Sci. Am. 99, 2439 (1996); C. Slotta and U. Heinz, Phys. Rev. D 58, 526 (1998).

[3] M.R. Andrews, C.G. Townsend, H.-J. Miesner, D.S. Durfee, D. M. Kurn, and W. Ketterle, Science 275, 637 (1997); Y. Castin and J. Dalibard, Phys. Rev. A 55, 4330 (1997); G. Baym, A.J. Leggett, and C.J. Pethick, to be published.

[4] G. Roland et al., (NA49 collaboration), Nucl. Phys. A638, 91c (1998); H. Appelhäuser et al., (NA49 collaboration), Phys. Lett. B459 (1999) 679.

[5] G. Baym, G. Friedman, and I. Sarcevic, Phys. Lett. 219B, 205 (1989).

[6] H. Heiselberg, G.A. Baym, B. Blättel, L.L. Frankfurt, and M. Strikman, Phys. Rev. Lett. 67, 2946 (1991); B. Blättel, G.A. Baym, L.L. Frankfurt, H. Heiselberg and M. Strikman, Nucl. Phys. A544, 479c (1992).

[7] G. Baym, B. Blättel, L. L. Frankfurt, H. Heiselberg, and M. Strikman, Phys. Rev. C 52, 1604 (1995).

[8] T. Åkesson et al. (Helios collaboration), Z. Phys. C38, 383 (1988).

[9] M. Gaździcki and S. Mrówczyński, Z. Phys. C54 127 (1992).

[10] S. Mrówczyński, Phys. Lett. B430, 9; B439, 6 (1998).

[11] M. Stephanov, K. Rajagopal, and E. Shuryak, Phy. Rev. Lett. 81, 4816 (1998); K. Rajagopal, hepth/9808348; M.A. Halasz, A.D. Jackson, R.E. Shrock, M.A. Stephanov, J.J.M. Verbaarschot, Phys. Rev. D 58, 96007 (1998)

[12] M. Stephanov, K. Rajagopal, and E. Shuryak, hep$p h / 9903292$.

[13] K. Werner, private communication.

[14] M. Gazdzicki \& O. Hansen, Nucl. Phys. A528 (1991) 754; W. Wroblewski, Acta Phys. Pol. B4 (1973) 857.

[15] J. Schukraft et al. (NA34 collaboration), Nucl. Phys A498, 79c (1989).

[16] T.K. Nayak (WA98 collaboration), private communication.

[17] H. Heiselberg and A. Levy, Phys. Rev. C (1999) (in press), nucl-th/9812034.

[18] G. Bertsch, Phys. Rev. Lett. 72, 2349 (1994).

[19] S. Jeon and V. Koch, nucl-th/9906074.

[20] H. Heiselberg, Phys. Lett. B379, 27 (1996).

[21] I.G. Bearden et al. (NA44 collaboration), Phys. Rev. Lett. 782080 (1997).

[22] H. Heiselberg and A.D. Jackson, Proc. Adv. in QCD, Minnesota, May 1998, nucl-th/9809013. 